\title{
Stärker auf Depression als Begleiterkrankung achten
}

\author{
Die Lebensqualität von Psoriasis-Patienten kann durch die \\ Erkrankung erheblich beeinträchtigt sein. Dabei berichten \\ Betroffene u a. von depressiven Symptomen und klinischen \\ Depressionen. E. A. Dowlatshahi et al. haben nun versucht, die \\ Prävalenz und das Risiko bei Psoriasis-Patienten abzuschätzen. \\ J Investig Dermatol 2014; 134: 1542-1551
}

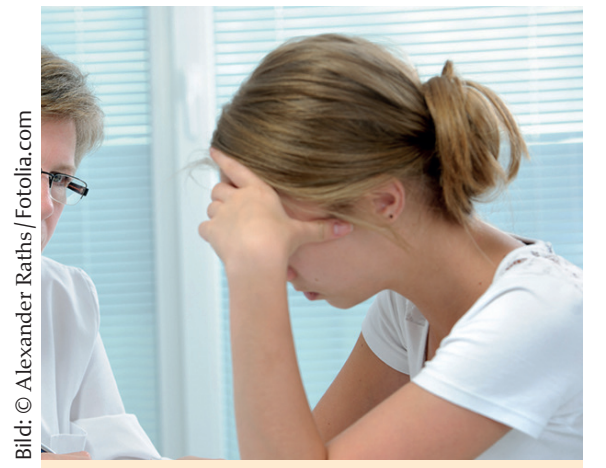

Bei Psoriasis-Patienten mit entsprechenden Symptomen sollte auch eine komorbide klinische Depression in Betracht gezogen werden.

Die bisher beschrieben Prävalenz von Depressionen bei Psoriasis-Patienten weist eine erhebliche Variabilität auf. Für ihre Analyse werteten die Autoren mit verschiedenen Techniken gemittelte Daten aus Fragebögen von Patienten mit depressiven Symptomen und klinischen Depressionen und der dazu publizierter Literatur aus. 98 Studien waren verfügbar. Die meisten Untersuchungen waren in großen Kliniken der Maximalversorgung ohne Kontrollgruppen durchgeführt worden, nur 26 Studien umfassten Patienten mit Psoriasis sowie gesunde Kontrollen.

Die Prävalenz depressiver Symptome nach Fragebogen-gestützten Untersuchungen lag bei $28 \%$ mit großen Unterschieden bei der Verwendung verschiedener Fragebögen. Die Prävalenz einer klinisch manifesten Depression unterschied sich ebenfalls je nach verwendeter Definition: Nach dem ICD-Code (International Classification of Diseases) betrug sie im Mittel 12\%, nach DSM-IV (Diagnostic and Statistical Manual of Mental Disorders IV) $19 \%$ und auf Basis der Verordnung von Antidepressiva $9 \%$.
Patienten mit Psoriasis wiesen im Vergleich zu gesunden Kontrollen deutlich mehr depressive Symptome auf (standardisierter mittlerer Unterschied [SMD] 1,16; 95\%-Konfidenzintervall [KI] 0,671,66). In den 8 analysierten populationsbasierten Studien ließ sich ein mind. 1,5-fach erhöhtes Risiko bei Patienten mit Psoriasis für eine Depression im Vergleich zu Kontrollen zeigen (OR=1,57; 95\%-KI 1,40-1,76). Die errechnete Prävalenz der Depression bei Psoriasis schwankte aber deutlich zwischen 2 und $10 \%$. Noch deutlicher als das Depressionsrisiko war die Wahrscheinlichkeit der Einnahme von Antidepressiva im Vergleich zu gesunden Kontrollen erhöht (OR=4,24, 95\%-KI 1,53-11,76). Das Risiko für Depressionen war bei Patienten mit Psoriasis unabhängig vom mittleren Alter und der Geschlechterverteilung in den Studien erhöht.

\section{Fazit}

Mehr als jeder 10. in den Studien untersuchte Patient mit Psoriasis litt unter einer klinischen Depression, doppelt so viele unter depressiven Symptomen. Dabei spielten sicher die Besonderheiten von Patientenkohorten an universitären Zentren eine Rolle. Zudem haben auch die verschiedenen Fragebögen und diagnostischen Kriterien einen großen Einfluss auf die Ergebnisse. Die Autoren empfehlen die Anwendung von Lebensqualitäts- und Depressionsfragebögen immer dann, wenn Patienten aufgrund ihrer Psoriasis stark beeinträchtigt erscheinen. Bei Verdacht auf eine klinische Depression sollten entsprechende Zeichen abgefragt und gegebenenfalls ein Psychiater hinzugezogen werden.

Friederike Klein, München
Forschung

\section{Neues Antibiotikum gegen MRSA}

Staphylococcus aureus ist der häufigste Verursacher von Krankenhausinfektionen in Deutschland. Beim Menschen kann der Erreger zu Wundinfektionen, Atemwegsentzündungen und Harnwegsinfektionen führen. Da das Bakterium gegenüber einer Vielzahl von Antibiotika resistent ist, wird es auch als multiresistenter oder methicillin-resistenter Staphylococcus aureus - kurz MRSA - bezeichnet. Den Wissenschaftlern um F. Surup ist es nun gelungen, 2 Substanzen aus den im Boden lebenden Myxobakterien zu isolieren, die hochwirksam gegen die Erreger sind. Ihre Ergebnisse wurden im Oktober vorab online in der Fachzeitschrift Angewandte Chemie International Edition veröffentlicht.

„Wir konnten zeigen, dass Disciformycin A und B hochaktiv gegen resistente Staphylokokken und andere Erreger sind. Außerdem konnten wir nachweisen, dass keine Kreuzresistenz gegenüber anderen eingesetzten Antibiotika vorliegt“, so die Forscher. „Sie wirken also nicht genauso wie bereits bekannte Antibiotika." Das gefundene Antibiotikum bekämpft gram-positive Bakterien, ohne den menschlichen oder tierischen Zellen zu schaden. Bevor die gefundenen Substanzen auch wirklich als Antibiotikum zum Einsatz kommen, stehen noch einige Schritte an: Zunächst muss die Produktion der Stoffe optimiert werden, um hinreichend Substanz zu erhalten. Anschließend folgen chemische Veränderungen des Wirkstoffkandidaten und die Überprüfung der pharmazeutischen Eigenschaften.

Außerdem versuchen die Forscher herauszufinden, wie die neu entdeckten Stoffe ihre Wirkung im Detail entfalten. Erst danach können Studien am Tiermodell folgen, die für den Einsatz als Medikament benötigt werden. Diese dienen vor allem dazu herauszufinden, wie toxisch die Wirkung der neuen Substanzen für den Körper ist. Im Anschluss muss das Molekül dann in einer Phase-I-Studie am Menschen getestet werden.

Nach einer Mitteilung des HelmholtzZentrums für Infektionsforschung, Braunschweig 